\title{
On the Nose
}

\section{Citation}

Abdalla, Marwah, Paul E. Sax, Arash Mostaghimi, Amy Leigh Miller, and Joseph Loscalzo. 2015. "On the Nose." Edited by Caren G. Solomon. New England Journal of Medicine 373 (10) (September 3): 955-961. doi:10.1056/nejmcps1315433.

\section{Published Version}

doi:10.1056/NEJMcps1315433

\section{Permanent link}

http://nrs.harvard.edu/urn-3:HUL.InstRepos:33785894

\section{Terms of Use}

This article was downloaded from Harvard University's DASH repository, and is made available under the terms and conditions applicable to Other Posted Material, as set forth at http:// nrs.harvard.edu/urn-3:HUL.InstRepos:dash.current.terms-of-use\#LAA

\section{Share Your Story}

The Harvard community has made this article openly available.

Please share how this access benefits you. Submit a story.

Accessibility 


\section{CLINICAL PROBLEM-SOLVING}

Caren G. Solomon, M.D., M.P.H., Editor

\section{On the Nose}

\section{Marwah Abdalla, M.D., M.P.H., Paul E. Sax, M.D., Arash Mostaghimi, M.D., M.P.A., Amy Leigh Miller, M.D., Ph.D., and Joseph Loscalzo, M.D., Ph.D.}

In this Journal feature, information about a real patient is presented in stages (boldface type) to an expert clinician, who responds to the information, sharing his or her reasoning with the reader (regular type). The authors' commentary follows.

A 67-year-old man presented to his primary care provider with a 3-day history of chest tightness, wheezing, coughing, and dyspnea. He reported a history of similar episodes that had been attributed to asthma, which was triggered by environmental allergens (mold, cats, grass, and ragweed). Although he had required hospitalization for asthma as a young man, his symptoms subsequently had been well controlled with inhaled triamcinolone, salmeterol, and loratadine, and he had had no major exacerbations for 20 years. He had rarely required his albuterol rescue inhaler. He reported no new allergen or environmental exposures, fevers, sputum production, hemoptysis, orthopnea, paroxysmal nocturnal dyspnea, edema, or weight loss. Pulmonary examination was unremarkable, with a normal respiratory rate and no wheezes; a radiograph of the chest was normal. He was prescribed prednisone to be tapered gradually over a 2-week period for presumed exacerbation of asthma.

Given a history of asthma and current symptoms that subjectively mimic those of prior asthma exacerbations, treatment of a presumed asthma exacerbation is reasonable. The absence of a clear trigger does not rule out asthma, particularly in a patient with multiple previously documented allergies. However, the long interval since his last exacerbation makes the diagnosis less certain.

His symptoms abated transiently but recurred shortly after the completion of the prednisone taper. He contacted his primary care provider, who prescribed another prednisone taper and provided prescriptions for refills of prednisone to treat any future exacerbations. Over the next several months, the patient reported that whenever his prednisone dose decreased below $20 \mathrm{mg}$ per day, the symptoms recurred. He occasionally took additional prednisone doses in a rescue fashion, at doses of up to $100 \mathrm{mg}$ per day. Peak flows were approximately 550 liters per minute when he was asymptomatic and $\mathbf{4 0 0}$ to $\mathbf{4 5 0}$ liters per minute when he was symptomatic. A protonpump inhibitor and a course of oral antibiotic agents were prescribed but had no effect. Fatigue, arthralgias, and pain in the left lower quadrant developed, and the patient lost weight.

The persistence of symptoms despite multiple prednisone courses is worrisome, as is the new constellation of symptoms. Although the history suggests a glucocorticoid-responsive process, I would not restrict the differential diagnosis according to glucocorticoid response. Indefinite use of glucocorticoids confers a risk of untoward consequences, including an increased risk of infection with prolonged use of prednisone at doses of $20 \mathrm{mg}$ per day or higher, and is particularly troubling in
From the Department of Medicine, Division of Cardiology, Columbia University Medical Center, New York (M.A.); and the Departments of Medicine (P.E.S., A.M., A.L.M., J.L.) and Dermatology (A.M.), Brigham and Women's Hospital and Harvard Medical School, Boston. Address reprint requests to Dr. Miller at the Department of Medicine, Brigham and Women's Hospital, 75 Francis St., Boston, MA 02115, or at almiller@partners.org.

N Engl J Med 2015;373:955-61. DOI: 10.1056/NEJMcps1315433 Copyright @ 2015 Massachusetts Medical Society. 
the absence of a clear diagnosis; I would taper the glucocorticoids. A careful review of the patient's travel history (recent and remote) and environmental exposures is warranted, and further imaging should be considered.

Four months after the initial presentation, the patient continued to have pulmonary and systemic symptoms, for which glucocorticoid therapy was continued. His physical examination remained unremarkable. Laboratory evaluation showed a hematocrit of $34.4 \%$ (baseline [1 year previously], $46.9 \%$ ), a white-cell count of 10,700 per cubic millimeter, and a platelet count of 569,000 per cubic millimeter. Computed tomography (CT) of the chest, abdomen, and pelvis revealed diverticulitis with a 6-cm abscess and opacification in the base of the left lung that was consistent with either a mass or an area of consolidation. The patient was advised to present to the emergency department for evaluation, and he did so 2 days after the CT. Repeat CT of the abdomen with the use of contrast material confirmed the presence of perforated diverticulitis with abscess and an incompletely imaged necrotic mass in the left lower lobe that was greater than $3 \mathrm{~cm}$ in diameter, with ground-glass opacification in the posterior aspect of both lower lobes. These findings prompted his admission to the hospital.

The patient's age places him at risk for diverticulitis, which is often polymicrobial; broad-spectrum antibiotic coverage is indicated. The only association between the diverticular abscess and his previous pulmonary symptoms may be the prednisone, which may have suppressed the clinical manifestations of diverticulitis, thereby contributing to the progression to perforation and abscess.

The patient's medical history included hypercholesterolemia, hypertension, colonic adenoma, benign prostatic hypertrophy, and chronic leg edema that resulted from vein stripping. At 18 years of age, he had had prolonged pharyngitis that was attributed to streptococcal infection, for which he had declined treatment for religious reasons. During that period, deep-vein thrombosis had developed while he was on bed rest. Additional medications at the time of admission included rosuvastatin, irbesartan, hydrochlorothiazide, and ezetimibe. $\mathrm{He}$ had no known drug allergies but could not take amlodipine and nifedipine because of side effects.
The patient worked as an actuary and lived with his wife and their dog. He smoked a pipe and had a remote history of cigarette use (10 packyears, with no cigarette use for more than four decades). He reported that he did not drink alcohol or use illicit drugs. His family history was negative for cancer and gastrointestinal disease. On admission, levofloxacin and metronidazole were started, and percutaneous abdominal drainage was performed. However, he declined consultation for consideration of lung biopsy, because he was reluctant to undergo further invasive procedures. Bacterial cultures grew more than 20 colonies of alpha-hemolytic streptococci (limited to the first quadrant) and mixed anaerobic flora. Fungal cultures were negative. The white-cell count was 14,430 per cubic millimeter at admission and declined to 11,560 per cubic millimeter at discharge 2 days later. The hematocrit and platelet values were unchanged from those at admission. He was discharged while taking oral antibiotics and with the abdominal drain in place; visiting nursing care was provided. He was encouraged to pursue pulmonary evaluation in the outpatient setting.

Imaging in this patient has revealed a necrotic lung lesion for which he has declined evaluation. I would have strongly counseled the patient that biopsy would probably enable diagnosis and the initiation of appropriate treatment, whereas delaying diagnosis and treatment could result in further progression and complications. Ultimately, however, as long as a patient is competent to make decisions, he has the right to decline.

A few days after the patient was discharged, a soft, mildly tender, violaceous nodule appeared on the right side of the tip of his nose and rapidly enlarged to $1.5 \mathrm{~cm}$ in diameter over the next 3 weeks. The patient then noted increasing edema in his lower legs bilaterally. Ultrasonography of the legs revealed deep-vein thromboses in the common femoral, superficial femoral, and popliteal veins bilaterally. He was hospitalized for further treatment and an expedited evaluation, which he agreed to pursue.

His bilateral thromboses may reflect a previously undiagnosed thrombophilia, given his history of deep-vein thrombosis. However, his pulmonary abnormalities may have conferred a predisposition to deep-vein thrombosis; both 
cancer and acute infection can increase the risk of venous thromboembolism.

Given the abnormal findings on chest imaging and his prolonged symptoms, further pulmonary evaluation is clearly indicated, but the new nasal lesion also warrants prompt attention. Given the extensive sun exposure of the nose, the rapid growth of the lesion, and tenderness on evaluation, a keratoacanthoma would be the most likely cancer. Other variants of squamous-cell carcinoma are less likely to grow as rapidly in an immunocompetent host. Basal-cell carcinoma is unlikely, given the violaceous color and rapid growth of this lesion. Dissemination of internal cancers to the skin, although uncommon, must be considered.

The nodule could also reflect an infectious process, which could involve bacterial, atypical mycobacterial, or fungal pathogens. The pulmonary and cutaneous findings in this patient could be explained by a number of infectious pathogens, including nocardia, Cryptococcus neoformans, Sporothrix schenckii, or one of the geographically constrained fungi, such as Histoplasma capsulatum and Blastomyces dermatitidis. Histoplasmosis rarely causes skin lesions in immunocompetent hosts; in this patient, the long-term glucocorticoid use may have been a predisposing factor. Rheumatologic processes that cause cutaneous and pulmonary abnormalities, such as sarcoidosis or granulomatosis with polyangiitis, are less likely.

Further history revealed that the patient was born in the Midwest and lived in Iowa, Wisconsin, and Illinois before moving to Massachusetts as a teenager. He had never traveled outside the United States. His most recent travel had been to Chicago, 2 months after symptom onset.

His travel history suggests that the geographically constrained fungi $H$. capsulatum and B. dermatitidis are considerations. Both these fungi are found in the Midwest.

The patient consented to hospitalization. On admission, he appeared well-nourished and in no acute distress. The temperature was $36.5^{\circ} \mathrm{C}$ $\left(97.7^{\circ} \mathrm{F}\right)$, the blood pressure $116 / 76 \mathrm{~mm} \mathrm{Hg}$, the pulse 90 beats per minute, the respiratory rate 16 breaths per minute, and the oxygen saturation $100 \%$ while the patient was breathing ambient air. The oropharynx was clear, without thrush or other lesions. The jugular venous pressure was not elevated. The lungs were clear to auscultation. Cardiovascular examination revealed a regular rate and rhythm without murmurs, rubs, or gallops. The abdomen was soft and nontender, with normal bowel sounds and without hepatosplenomegaly. There was no cervical, supraclavicular, axillary, or inguinal lymphadenopathy. The hands and feet were warm. There was $2+$ pitting edema in the lower legs. Skin examination revealed Fitzpatrick type II skin (burns easily, tans minimally) with a $1.5-\mathrm{cm}$ violaceous nodule over his right nasal sidewall (Fig. 1). The nodule was fluctuant with a scaly surface and was tender to deep palpation, expressing some mucopurulent discharge with manipulation.

Although the nasal lesion could be a squamouscell carcinoma, such lesions are usually firm. In addition, the presence of mucopurulent discharge suggests infection rather than cancer. At this time, an expedited laboratory workup should be conducted, including testing for serum cryptococcal antigen, antinuclear antibody,

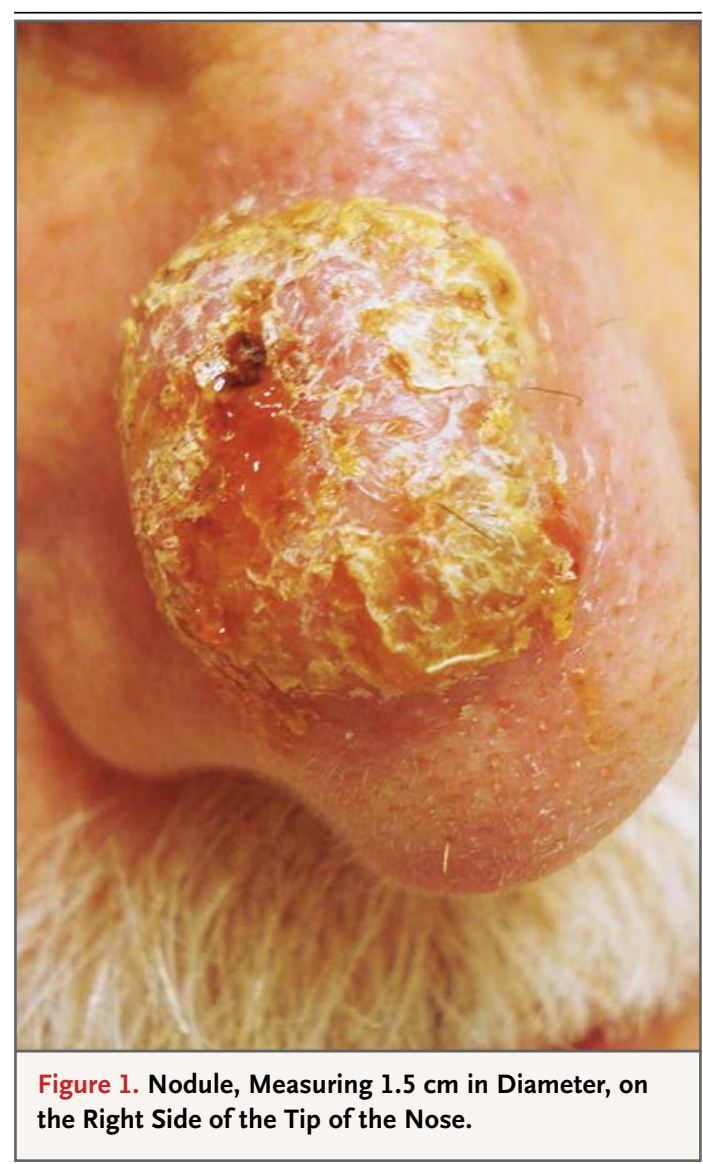


antineutrophil cytoplasmic antibody, and human immunodeficiency virus (HIV); in addition, a nasal biopsy should be performed and bronchoscopy should be considered.

The white-cell count was $\mathbf{4 8 0 0}$ per cubic millimeter $(71 \%$ polymorphonuclear cells, $20 \%$ lymphocytes, $7 \%$ monocytes, and $2 \%$ eosinophils), the hematocrit $36 \%$ with a mean corpuscular volume of $96.2 \mathrm{fl}$, and the platelet count 363,000 per cubic millimeter. A comprehensive metabolic panel and coagulation studies were normal. The iron level was $57 \mu \mathrm{g}$ per deciliter $(\mathbf{1 0} \mu \mathrm{mol}$ per liter; reference range, 37 to $158 \mu$ g per deciliter [ 7 to $28 \mu \mathrm{mol}$ per liter]), the total iron-binding capacity $231 \mu \mathrm{g}$ per deciliter (41 $\mu \mathrm{mol}$ per liter; reference range, 250 to $450 \mu \mathrm{g}$ per deciliter [ 45 to $81 \mu \mathrm{mol}$ per liter]), and the ferritin level $873 \mathrm{ng}$ per milliliter (reference range, 10 to 170). Testing for HIV antibody was negative. Gram's staining of discharge from the nasal lesion showed 1 to 5 polymorphonuclear cells per low-power field and no organisms. Culture grew coagulase-negative staphylococcus. A hypercoagulation workup (including assessment of activated protein $\mathrm{C}$ resistance, measurement of levels of protein C, protein S, homocysteine, antithrombin III, and lupus anticoagulant, and testing for the prothrombin gene mutation) was negative.

The anemia evaluation suggests a chronic inflammatory process. The results of the nasallesion culture do not rule out other organisms. In fact, most of the previously mentioned fungal organisms and nocardia require special media or additional time to grow. A wet mount or a preparation with potassium hydroxide could identify fungal organisms. On a biopsy specimen, methenamine silver stain would be the preferred stain to identify fungi. If yeast forms

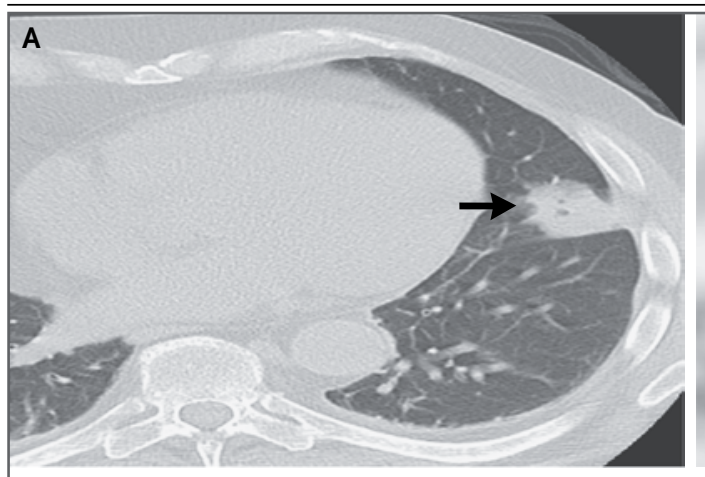

\section{B}

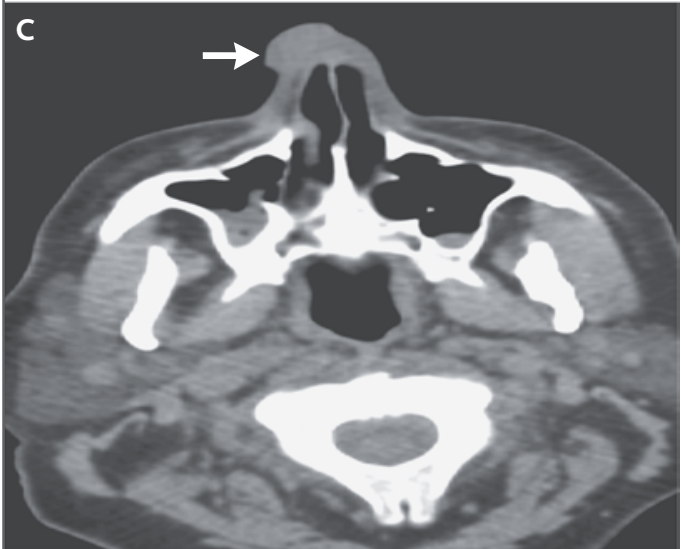

\section{D}

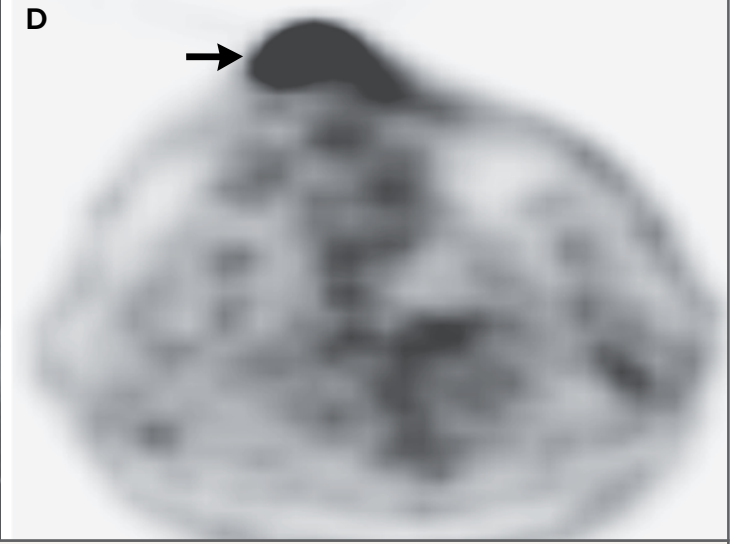

Figure 2. CT Images of the Chest and Head.

A positron-emission tomographic (PET)-computed tomographic (CT) scan of the chest showed a lingular mass measuring $4.5 \mathrm{~cm}$ by $2.7 \mathrm{~cm}$ (Panel A, arrow), with moderate ${ }^{18} \mathrm{~F}$-fluorodeoxyglucose (FDG) uptake (Panel B, arrow). A CT image from a PET-CT scan of the head showed a right nasal lesion (Panel C, arrow), with intense FDG uptake in the lesion (Panel D, arrow). 
are found, the mucicarmine stain will identify the capsular material of $C$. neoformans. The negative result on Gram's staining argues against nocardia, which is a thin, branching, grampositive bacillus. When a disseminated fungal infection is suspected, the most useful noninvasive diagnostic tests are serum testing for cryptococcal antigen and urine testing for histoplasmosis antigen.

A pulmonary CT angiogram showed a segmental pulmonary embolism in the right lower lobe and a mass in the left lingula measuring $4.5 \mathrm{~cm}$ by $2.7 \mathrm{~cm}$, without adenopathy. Enoxaparin was started. A positron-emission tomographic (PET) scan showed moderate ${ }^{18} \mathrm{~F}$-fluorodeoxyglucose (FDG) avidity in the lung mass and intense FDG uptake in the right nasal lesion (Fig. 2).

The PET scan is expensive and is of limited usefulness here. Moderate FDG avidity can be seen in both malignant and infectious processes. I suspect a disseminated infection, which is most likely to be fungal, given the patient's previous residence in an area in which histoplasmosis and blastomycosis are endemic, the lack of lymphadenopathy, and the rapidly enlarging nasal nodule. A biopsy of the nasal lesion may provide a tissuebased diagnosis.

The patient consented to excision of the nasal lesion. Pathological findings showed a well-differentiated keratoacanthoma-like squamous-cell carcinoma.

As noted previously, fungal processes can be missed on initial staining. I would review the nasal-biopsy results, ensuring that proper stains (in particular, methenamine silver and acid-fast stains) were used. If these tests were negative or could not be performed, a lung biopsy would be indicated.

A fine-needle aspiration of the lung revealed necrotizing granulomatous inflammation. Gomori methenamine silver staining revealed fungal organisms consistent with blastomycosis (Fig. 3). Mucicarmine, acid-fast bacilli, Gram's, and Fontana-Masson stains were negative. On reexamination of the nasal-biopsy specimen, periodic acid-Schiff staining confirmed the presence of blastomycosis with associated massive pseudo- epitheliomatous hyperplasia that mimicked squamous-cell carcinoma (Fig. 4). Urine testing for blastomyces antigen was positive at 4.3 units on enzyme immunoassay. The patient began receiving a 6-month course of itraconazole. His symptoms almost completely resolved within 1 month, and clinically significant regression of the pulmonary parenchymal abnormalities was observed after 3 months. The blastomyces antigen level on urine testing slowly declined and was undetectable at the end of treatment. One year after his diagnosis, he remained symptom-free.

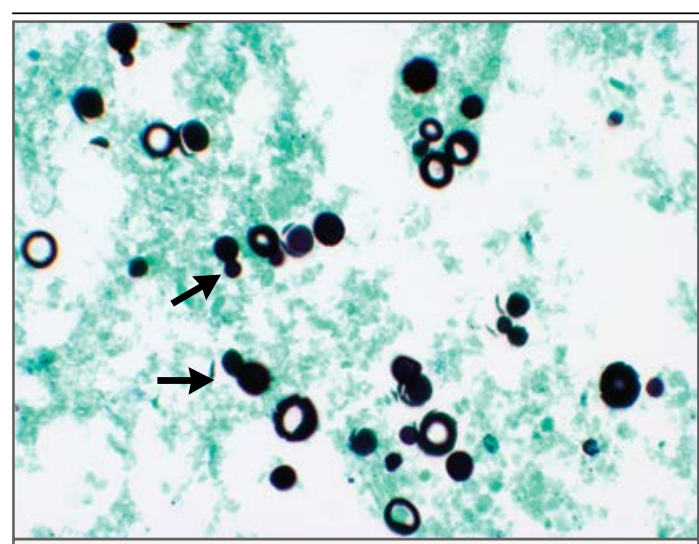

Figure 3. Fine-Needle Aspirate of the Lung.

A fine-needle aspirate of the lung stained with Gomori methenamine silver showed broad-based budding yeast (arrows) with features characteristic of Blastomyces dermatitidis.

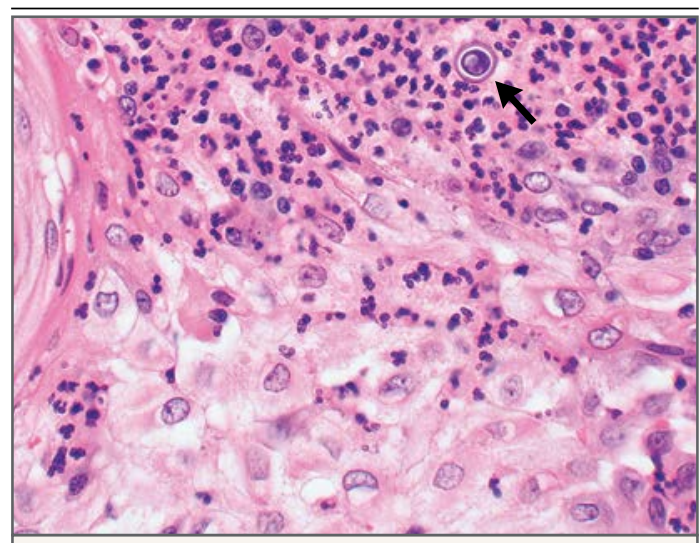

Figure 4. Nasal-Biopsy Specimen.

A periodic acid-Schiff stained specimen from the nasal biopsy showed a B. dermatitidis yeast cell (arrow). 
COMMENTARY

Diagnosis of blastomycosis is often delayed because of the low clinical suspicion at the patient's presentation and the range of associated clinical symptoms. In this patient, the respiratory symptoms, pulmonary imaging, and travel history could have prompted earlier diagnosis, but it was not until the nasal lesion appeared that the travel history was obtained.

Blastomycosis is caused by the thermally dimorphic fungus $B$. dermatitidis. In North Ameri$\mathrm{ca}, \mathrm{B}$. dermatitidis is endemic in regions bordering the Great Lakes and the Saint Lawrence Seaway, as well as in the Mississippi and Ohio River valleys, the Canadian province of Manitoba, and the southeastern United States. ${ }^{1}$ B. dermatitidis causes disease when conidia are inhaled and converted to budding yeast at body temperature. ${ }^{2}$

Because exposure occurs primarily by means of inhalation, pulmonary involvement is the most common disease manifestation. Pulmonary blastomycosis can be asymptomatic, acute, or chronic and can mimic cancer or infection with pyogenic bacteria, Mycobacterium tuberculosis, or fungi. ${ }^{2}$ Symptoms range from a mild influenza-like illness to cough with sputum production and dyspnea; in rare instances, infection can lead to the acute respiratory distress syndrome, most commonly in patients who have a history of diabetes and those who have had prior antimicrobial therapy. ${ }^{2}$

Radiographic manifestations of pulmonary blastomycosis include focal masses, nodules, interstitial disease, air-space consolidation, and cavitary lesions. ${ }^{3}$ Patients with acute blastomycosis tend to have air-space consolidation on imaging, whereas those with chronic infection may present with mass lesions. Pleural effusions and lymphadenopathy are rare manifestations. ${ }^{3}$

Hematogenous dissemination can lead to extrapulmonary blastomycosis. The skin is the most common extrapulmonary site; cutaneous lesions occur in $80 \%$ of patients with pulmonary blastomycosis ${ }^{4}$ and, in contrast to lesions associated with histoplasmosis, commonly occur in immunocompetent as well as immunosuppressed hosts. Although primary cutaneous blastomycosis is rare, it can occur by means of direct inoculation with B. dermatitidis. Both primary and secondary cutaneous blastomycosis can manifest as papulopustules, ulcers, nodules, or verrucous lesions that can be mistaken for squamous-cell carcinoma. ${ }^{1,5}$ Histopathologically, these lesions often show pseudoepitheliomatous hyperplasia with intraepidermal microabscesses and occasional round yeast forms with broad-based budding. ${ }^{5}$ Other sites of dissemination include the genitourinary tract, bone, and central nervous system. The risk of dissemination is increased among pregnant women and immunosuppressed persons, including recipients of solid-organ transplants ${ }^{1,6}$ patients with advanced HIV disease, ${ }^{1,6}$ and patients with prolonged glucocorticoid use. ${ }^{6}$

Methenamine silver or periodic acid-Schiff staining and direct visualization of $B$. dermatitidis in tissue specimens is the usual diagnostic method, especially in extrapulmonary disease. ${ }^{7}$ B. dermatitidis can also be cultured from sputum and bronchial washings. Urine testing for $B$. dermatitidis antigen is an immunoassay with a sensitivity of $93 \%$ and specificity of $79 \%$ for blastomycosis. ${ }^{8}$ However, there is cross-reactivity with several other fungi. ${ }^{7,9}$

Treatment of moderate-to-severe pulmonary or disseminated blastomycosis consists of amphotericin B for 1 to 2 weeks until symptoms abate, followed by itraconazole therapy for 6 to 12 months. For mild-to-moderate disease, including disseminated disease that is limited to the skin, monotherapy with itraconazole for 6 to 12 months is recommended. Serum levels of itraconazole should be assessed after 2 weeks to ensure adequate levels. ${ }^{7}$

This case highlights the hazards of anchoring on an initial diagnosis despite the lack of a response to therapy, the importance of reviewing primary data, including pathological specimens, and the importance of exposure history. Although the patient's reluctance to undergo invasive evaluation complicated the diagnostic process in this case, the history of residence in an area where blastomycosis is endemic was key to focusing the differential diagnosis. Although the evaluation ultimately led to the correct diagnosis and effective treatment, earlier diagnosis with less-invasive testing would have been possible.

No potential conflict of interest relevant to this article was reported.

Disclosure forms provided by the authors are available with the full text of this article at NEJM.org.

We thank Drs. Jeffrey F. Krane and Rosalynn Nazarian for clinical input. 
REFERENCES

1. McKinnell JA, Pappas PG. Blastomycosis: new insights into diagnosis, prevention, and treatment. Clin Chest Med 2009;30:227-39.

2. Kralt D, Light B, Cheang $M$, et al. Clinical characteristics and outcomes in patients with pulmonary blastomycosis Mycopathologia 2009;167:115-24.

3. Fang W, Washington L, Kumar N. Imaging manifestations of blastomycosis: a pulmonary infection with potential dissemination. Radiographics 2007;27:641-55.

4. Medoff G, Kobayashi GS. Systemic fungal infections: an overview. Hosp Pract (Off Ed) 1991;26:41-52.

5. Mason AR, Cortes GY, Cook J, Maize JC, Thiers BH. Cutaneous blastomycosis: a diagnostic challenge. Int J Dermatol 2008;47:824-30.

6. Pappas PG, Threlkeld MG, Bedsole GD, Cleveland KO, Gelfand MS, Dismukes WE. Blastomycosis in immunocompromised patients. Medicine (Baltimore) 1993;72:311-25.

7. Chapman SW, Dismukes WE, Proia LA, et al. Clinical practice guidelines for the management of blastomycosis: 2008 update by the Infectious Diseases Society of America. Clin Infect Dis 2008;46:1801-12. 8. Bariola JR, Hage CA, Durkin M, et al. Detection of Blastomyces dermatitidis antigen in patients with newly diagnosed blastomycosis. Diagn Microbiol Infect Dis 2011;69:187-91.

9. Durkin M, Witt J, Lemonte A, Wheat B, Connolly P. Antigen assay with the potential to aid in diagnosis of blastomycosis. J Clin Microbiol 2004;42:4873-5.

Copyright (c) 2015 Massachusetts Medical Society.

CLINICAL PROBLEM-SOLVING SERIES

The Journal welcomes submissions of manuscripts for the Clinical Problem-Solving series. This regular feature considers the step-by-step process of clinical decision making. For more information, please see authors.NEJM.org. 